\title{
Um olhar sobre o papel do pai na compreensão emocional das crianças: Os estilos parentais e práticas de socialização das emoções negativas
}

\author{
Mara Chora ${ }^{1}$, Lígia Monteiro ${ }^{1,2}$, Madalena Ramos $^{1,3} \&$ Rita Amaral $^{1}$ \\ ${ }^{1}$ ISCTE-Instituto Universitário de Lisboa \\ ${ }^{2}$ CIS-IUL \\ ${ }^{3}$ CIES-IUL
}

\begin{abstract}
Resumo: Os pais são figuras centrais no desenvolvimento emocional da criança durante os seus primeiros anos de vida, com a literatura a focar-se, essencialmente, no impacto da mãe na aquisição e desenvolvimento da compreensão emocional (CE), apesar de evidências recentes indicarem a importância que as práticas paternas poderão ter neste domínio. 0 presente estudo analisou o efeito dos estilos parentais e das práticas de socialização das emoções negativas do pai na CE das crianças, numa amostra de 75 pais e crianças portuguesas em idade pré-escolar. Os pais reportaram os seus estilos parentais e práticas através dos questionários PSDQ e CCNES, respetivamente. A CE foi avaliada pelo TEC. Verificou-se uma associação negativa entre a CE e o estilo permissivo e as reações de perturbação, e uma associação positiva com a idade da criança. Os resultados são discutidos equacionando características dos estilos e práticas paternas e o seu possível impacto na CE da criança.
\end{abstract}

Palavras-chave: Pai; estilos parentais; práticas de socialização emocional; compreensão emocional; préescolar.

The role of the father in children's emotional comprehension: Parenting styles and emotion socialization practices of negative emotions: Parents are central figures in children's emotional development during the first years of life. The core literature has focused mainly on the role that mothers have on children's emotional comprehension (EC), yet new evidence supports the notion that fathers practices may also play a role in this domain. The present study analysed the effects of father's parenting styles and socialization practices of negative emotions on children's EC, using a sample of 75 fathers and their Portuguese preschool age children. Fathers reported on their parenting styles and practices using the PSDQ and the CCNES questionnaires. Children's EC was analysed with the TEC. Negative associations were found between children's EC and father's permissive style and distress reactions. A positive association between EC and child's age was also found. Results were discussed in terms of father's parenting styles and practices characteristics and their potential impact on children's emotional comprehension.

Keywords: Father; emotion comprehension; parenting styles; emotion socialization practices; preschool.

Nas últimas décadas, o interesse sobre a importância e significado do papel do pai nas dinâmicas familiares e, em particular, nos cuidados e desenvolvimento da criança tem vindo a aumentar, não só por parte dos investigadores, mas também por políticos e decisores e pela sociedade em geral (Lamb, 2010; Wilson \& Prior, 2011). Esta mudança não pode ser, no entanto, dissociada do contexto macro, nomeadamente, das mudanças económicas e sociais verificadas ao nível do mercado de trabalho, das estruturas familiares e do paradigma de igualdade de géneros (Tamis-LeMonda \& Cabrera, 2002).

A família é o contexto primário de cuidados e afetos da criança, onde se estabelecem as relações iniciais e onde as figuras parentais desempenham diversas funções, entre as quais de socialização da criança (Denham, 1998; Parke et al., 2006). Neste âmbito, a literatura tem vindo a analisar de que modo a qualidade da parentalidade tem impacto no desenvolvimento, desde os primeiros anos de vida, e em diferentes domínios do desenvolvimento (Eisenberg, Cumberland, \& Spinrad, 1998; Halpenny, Nixon, \& Watson, 2010; Lamb, 2010). Tal como noutras áreas de estudo, mas com particular relevância no domínio socioemocional, a maioria da investigação centra-se na figura materna, colocando em segundo plano outras figuras como o pai, pelo que o presente estudo visa contribuir para colmatar esta limitação.

Uma das abordagens mais comuns na análise da parentalidade, ou do clima emocional fornecido aos filhos, é baseada nos estilos parentais. Estes referem-se ao conjunto de atitudes relativas à criança,

${ }^{1}$ Morada para correspondência: Lígia Monteiro; Avenida das Forças Armadas (Ala Autónoma, gabinete 101), 1649-026 Lisboa, Portugal. Email: ligia.monteiro@iscte-iul.pt. Este trabalho teve o apoio da Fundação para a Ciência e Tecnologia (FCT) em Portugal, enquadrado no financiamento PEST (UID/PSI/03125/2013). 
transmitidas através de comportamentos dos pais em interação com a mesma (e.g., práticas parentais, expressão emocional, gestos) (Darling \& Steinberg, 1993). Na tipologia clássica definida por Baumrind (1966) são identificados três estilos: o autoritativo, o autoritário e o permissivo. 0 estilo autoritativo é marcado pela responsividade e suporte às necessidades da criança, definição clara de regras e limites, boa capacidade de comunicação, onde é discutida e avaliada a perspetiva da criança. 0 estilo autoritário é definido pelo baixo suporte e elevada punição como forma de controlo dos comportamentos e atitudes da criança, restringindo a sua autonomia e expressão emocional e comportamental. Por fim, o estilo permissivo é caracterizado pela elevada responsividade e aceitação do ponto de vista da criança, mas por uma baixa imposição de regras e limites, evitando o cuidador o controlo e o assumir de um papel ativo na orientação do comportamento da mesma. Estes estilos são caracterizados por comportamentos que visam atender a objetivos específicos de socialização (Darling \& Steinberg, 1993), nomeadamente, da aprendizagem de comportamentos que resultam de experiências emocionais, observação da linguagem corporal e das interações entre as pessoas ou das respostas dos cuidadores às necessidades e emoções da criança (Halberstadt \& Lozada, 2011; Parke et al., 2006).

No contexto das emoções, as figuras parentais recorrem a práticas de socialização, como a modelagem ou a expressividade emocional, fornecendo à criança pistas sobre a natureza, causas, comportamentos e vocabulário associados a uma emoção. Tais práticas podem surgir aliadas à exploração dos estados emocionais com recurso ao diálogo e à resposta emocional e comportamental dos cuidadores face à emoção da criança. As respostas podem ser de contingência gratificante, i.e., reações de suporte, ou de contingência de punição, i.e., reforço negativo ou rejeição das emoções. Espera-se, pois, que estas estejam relacionadas com o desenvolvimento emocional das crianças (Denham, 1998; Denham, Basset, \& Wyatt, 2007).

No domínio emocional, a compreensão das emoções assume importância central por permitir ao indivíduo ajustar as suas interações com os outros (e.g., familiares, pares, professores) em diferentes contextos (Belacchi \& Farina, 2010). Especificamente, a compreensão emocional implica a capacidade de identificar uma expressão (e.g., facial) associada a um estado emocional, o conhecimento das causas e consequências das emoções, e a resposta apropriada à expressão emocional do outro. Engloba, ainda, a capacidade de nomear expressões emocionais (verbais e não verbais); a utilização de linguagem específica para descrever as experiências emocionais; o reconhecimento de que estas podem diferir entre indivíduos; o conhecimento da existência de estratégias de regulação da emoção; e a perceção de que duas emoções podem coexistir, mesmo sendo de valências distintas (Denham, 1998; Denham et al., 2003).

Pons, Harris e De Rosnay (2004) desenvolveram um modelo explicativo sobre o desenvolvimento da compreensão emocional, com início nos anos pré-escolares, que contempla três fases de nível de complexidade crescente, cada uma com três competências específicas. A primeira fase, denominada de Externa (cerca dos 5 anos), está relacionada com a compreensão das causas situacionais, a expressão da emoção e os acontecimentos ou objetos que funcionam como suscitadores da mesma. A segunda fase, a Mental (cerca dos 7 anos), é caracterizada pela compreensão da natureza das emoções, ou seja, a relação destas com as crenças e os desejos de cada um e a capacidade de distinguir entre a emoção que é sentida e a que é expressa. Por fim, a fase Reflexiva (cerca dos 9-11 anos), caracteriza-se pela capacidade de pensar sobre uma situação a partir de diferentes pontos de vista e identificar as emoções subjacentes a cada um, bem como regular a sua emoção recorrendo a estratégias cognitivas. Diversos estudos com amostras anglosaxónicas (e.g., Molina, Bulgarelli, Henning, \& Ashersleben, 2014), da Europa Latina (Belacchi \& Farina, 2010; Sagone \& Caroli, 2014) e de Portugal (Santos, 2012; Rocha et al., 2013) confirmam esta sequência, ainda que o surgimento de algumas competências possa não corresponder à ordem exata formulada pelo modelo. No estudo de Rocha e colaboradores (2013), com crianças em idade escolar, o conhecimento de estratégias de regulação emocional obteve uma percentagem de sucesso superior à capacidade de identificar que as emoções podem ser ocultadas por outra expressão facial. Também a compreensão das emoções associadas às crenças obteve uma percentagem de sucesso superior à da capacidade de compreender que uma situação passada pode suscitar uma emoção. Silva (2012) verificou, para a mesma faixa etária, que as crianças apresentavam um baixo desempenho no conhecimento das emoções associadas aos desejos e crenças. Ainda assim, e de uma forma geral, os resultados encontrados na literatura são consistentes e indicadores de que a idade da criança é um fator relevante na capacidade de compreensão emocional. A variabilidade verificada na ordem de desenvolvimento das diversas competências poderá estar relacionada com processos de socialização específicos das culturas de onde as amostras eram provenientes (Halberstadt \& Lozada, 2011). Relativamente ao sexo, a investigação tendencialmente não reporta diferenças entre os rapazes e as raparigas, em idade pré-escolar, quanto à capacidade de compreensão emocional (e.g., Belacchi \& Farina, 2010; Ornaghi, Grazzani, Cherubin, Conte, \& Piralli, 2015).

Os anos pré-escolares são fundamentais na aquisição e desenvolvimento das competências socioemocionais (Denham, 1998) e o papel do tipo e qualidade dos cuidados parentais assumem particular 
relevância neste mesmo período (e.g., Denham, Mitchell-Copeland, Strandberg, Auerbach, \& Blair, 1997). Embora se possa encontrar alguma variabilidade sociocultural, de modo geral, crianças cujas figuras parentais apresentam um estilo autoritativo apresentam melhores competências cognitivas (e.g., Bibi, Chaudhry, Awan, \& Tariq, 2013; Leung, Lo, Tsang, Chan, \& Kung, 2016) e sociais, bem como, menos problemas comportamentais (e.g., Bibi et al., 2013; Reed, 2015; Taleb, 2013). Os estilos autoritário e permissivo estão, geralmente, associados a menor rendimento escolar (e.g., Baumrind, 1966; Reed, 2015), mais problemas comportamentais (e.g., Jia, Wang, \& Shi, 2014) e menores competências cognitivas (e.g., Leung et al., 2016) e sociais (e.g., Taleb, 2013).

A investigação relativa às emoções, com base em comportamentos parentais, está essencialmente centrada na figura materna (Denham et al., 2007; Root \& Rubin, 2010) e a maioria dos estudos que incluem o pai, não fazem distinção entre ambos (e.g., Denham et al., 1997). Durante as idades pré-escolares, os comportamentos da mãe característicos do estilo autoritativo, como, por exemplo, o fornecer conforto ou explicar como um comportamento afeta as outras pessoas, estão positivamente associados com a capacidade da criança em nomear, reconhecer e compreender que a emoção depende dos desejos (Bennet, Bendersky, \& Lewis, 2005; Fabes, Poulin, Eisenberg, \& Madden-Derdich, 2002). Por outro lado, os comportamentos específicos do estilo autoritário e permissivo, como o ignorar ou evitar a criança, castigála ou retirar privilégios, encontram-se negativamente associados à capacidade de reconhecimento, nomeação e conhecimento das situações que causam a emoção (Bennet et al., 2005; Fabes et al., 2002; Sullivan, Carmody, \& Lewis, 2010). Bennet e colaboradores (2005) encontraram uma associação positiva e significativa entre a parentalidade positiva da mãe e a compreensão emocional da criança aos 4 anos. No entanto, verificaram que esta competência não era prevista pela parentalidade (positiva e negativa) da mãe. No estudo de Vinden (2001), que avaliava a componente específica da compreensão da falsa crença, não foram encontradas associações entre os estilos parentais maternos e a compreensão emocional.

Quando ambas as figuras parentais recorrem a estratégias de suporte encorajando a expressão emocional da criança, esta apresenta um maior ajuste psicossocial, utilização de formas adequadas de lidar com as emoções e níveis mais elevados de competência emocional ao nível da regulação, expressão e compreensão (Denham, 1998; Denham et al., 2007; Root \& Rubin, 2010). Pais e mães que ignoram ou punem as emoções da criança, demonstram pouco interesse no que esta tenta comunicar, mostram-se ansiosos ou irritados com a situação emocional, não analisando o significado da mesma, nem contribuindo para a resolução do problema, dificultam o desenvolvimento socioemocional da criança e o seu ajustamento social (Gottman \& DeClaire, 1997). Os cuidadores, ao exercerem maior controlo sobre as emoções negativas e não fornecendo suporte à criança, aumentam a probabilidade de esta apresentar um risco acrescido de desenvolvimento de problemas de externalização (e.g., agressividade) e de internalização (e.g., ansiedade). No entanto, estratégias de minimização emocional, quando levadas a cabo num ambiente afetivo de qualidade, podem não ser percecionadas pela criança como desvalorização da sua emoção, mas sim da situação em si (Friedlmeier, Corapci, \& Cole, 2011).

Denham e colaboradores (1997) verificaram que respostas mais adequadas de ambos os pais ao afeto positivo e à angústia da criança em idade pré-escolar contribuíam para um bom desenvolvimento ao nível da compreensão emocional. Figuras parentais que sejam mais positivas em situações desafiantes, com respostas de suporte e aceitação, permitem à criança uma melhor aprendizagem sobre as emoções, o que se reflete na sua competência social, ao promover respostas pró-sociais em relação aos pares. Resultados semelhantes foram encontrados após avaliação de um programa de intervenção que tinha como objetivos ensinar os cuidadores a reconhecer e a valorizar as emoções da criança em idade pré-escolar (Havighurst, Wilson, Harley, Prior, \& Kehoe, 2010). Em contrapartida, pais e mães que reforçam negativamente ou rejeitam as emoções expressas pela criança, por meios físicos, verbais ou comportamentais, têm um impacto negativo na compreensão emocional, especialmente, em crianças até aos 4 anos (Denham et al., 1997). Por outro lado, Guajardo, Snyder e Petersen (2009) não encontraram associações entre comportamentos parentais negativos (i.e., intrusividade na interação ou inconsistência parental) e a compreensão emocional, particularmente, na nomeação e conhecimento das causas de uma emoção.

Em suma, apesar da maioria da investigação ser relativa à mãe, o padrão de resultados encontrado na literatura vai no sentido de que as reações negativas ou de baixo suporte estão associadas a baixos níveis de competência emocional, enquanto as positivas ou de elevado suporte fornecem à criança meios de compreensão do pensamento e comportamentos subjacentes às situações de carga emocional. Através das reações às emoções negativas da criança, os cuidadores transmitem formas específicas de lidar com as mesmas, o que se insere em objetivos de socialização mais latos. Assim, a relação entre o contexto de socialização, i.e., estilo parental, e a compreensão emocional poderá ser moderada através de algumas variáveis parentais características das práticas de socialização das emoções, nomeadamente a valência, tipo e intensidade das emoções transmitidas e a adequação das respostas e comportamentos emocionais (Eisenberg et al., 1998). 
Ambos os pais desempenham um papel fundamental na socialização e desenvolvimento da criança, existindo estudos (e.g., Figueiredo, Mateus, Osório, \& Martins, 2014) que apontam para comportamentos semelhantes nas suas interações com os filhos, como encorajar à exploração do ambiente e responder aos seus sorrisos e choros. Porém, existe evidência de que comportamentos maternos e paternos tendem a ocorrer em contextos distintos, encontrando-se algumas diferenças entre os comportamentos de mães e pais, ao nível da socialização das emoções. Comparativamente com a mãe, o pai tende a fornecer mais suporte à ansiedade e a reportar mais respostas de aversão face à expressão de desapontamento dos rapazes, enquanto com as raparigas tende a pedir menos informação acerca das suas emoções. Não se verificam diferenças nas reações de ambos os pais face à expressão de zanga (Root \& Rubin, 2010).

Mazzone e Nader-Grosbois (2016), numa amostra de crianças em idade pré-escolar, verificaram que para as emoções negativas o pai recorre maioritariamente a estratégias de suporte (reações centradas nas emoções e problema, e encorajamento expressivo) e a reações de minimização, não tendo sido encontradas associações com o conhecimento emocional da criança. McElwain, Halberstadt e Volling (2007) encontraram um padrão de comportamentos semelhante, com o pai a recorrer maioritariamente a reações de suporte às emoções de crianças entre os 5 e os 6 anos, comparativamente com a demonstração de reações negativas por parte do mesmo progenitor. Quando o pai apresenta maior suporte, a criança revela maior capacidade de compreensão da emoção associada à falsa crença, mas não da compreensão da possibilidade de sentir várias emoções na mesma situação. As reações negativas ou de baixo suporte, por seu turno, não estão relacionadas com a compreensão emocional (McElwain et al., 2007). A socialização paterna das emoções, com respostas maioritariamente de suporte, parece contribuir para o conhecimento de situações e expressões emocionais em crianças de 4 anos, sendo um preditor da compreensão da possibilidade de esconder estados emocionais aos 5 anos (Denham \& Kochanoff, 2002).

Uma das questões fundamentais que se coloca quando analisamos a figura paterna remete para o contexto de interações que parece, tradicionalmente, caracterizar a relação pai-criança, em particular na idade pré-escolar. Embora se venha a assistir a um maior envolvimento do homem nos cuidados à criança, estudos com amostras portuguesas indicam que o pai participa mais em atividades de brincadeira/lúdicas, sendo as atividades de cuidados (e.g., dar banho e organizar as tarefas do dia a dia) essencialmente asseguradas pela mãe, com o pai a assumir um papel de apoio (Monteiro et al., 2010; Monteiro, Veríssimo, Santos, \& Vaughn, 2008; Perista, Carsoso, Brázia, Abrantes, \& Perista, 2016). Apesar de uma tendência positiva para a partilha de atividades e rotinas com a mãe, a brincadeira parece permanecer o contexto em que o pai se sente mais à vontade para fornecer à criança suporte afetivo, estimulação cognitiva e aprendizagens sociais (Monteiro et al., 2008), ocorrendo a socialização das emoções, também, nesse mesmo contexto. 0 estilo de interação e de brincadeiras mais físicas e estimulantes contribui para que a criança aprenda a interpretar e utilizar os sinais emocionais na regulação do comportamento social com os outros (Parke et al., 2006). O pai tende a promover maior desafio físico e cognitivo nas interações, explorando os seus aspetos emocionais e as suas causas e consequências, facilitando a compreensão das emoções (Lamb, 2010).

Dada a investigação sobre o desenvolvimento socioemocional se centrar, essencialmente, nas características da criança e nas práticas de socialização e estilos maternos (e.g., Bennet et al., 2005; Fabes et al., 2002; Guajardo et al., 2009; Sullivan et al., 2010), o presente estudo insere-se no esforço de um maior conhecimento sobre o papel do pai no domínio emocional da criança, analisando as relações entre os estilos parentais e práticas paternas de socialização das emoções negativas da criança e a compreensão emocional da criança, controlando a sua idade e sexo. Espera-se que: 1) o estilo parental autoritativo do pai esteja positiva e significativamente associado com a compreensão emocional global da criança, enquanto que os estilos autoritário e permissivo estejam negativamente associados com a mesma; 2) as práticas de socialização positivas estejam positiva e significativamente associadas com a compreensão emocional, e as práticas de socialização negativas estejam negativamente associadas à compreensão emocional. Testaramse, ainda, diferentes modelos de regressão hierárquica, no sentido de analisar quais as variáveis preditoras da compreensão emocional das crianças.

\section{MÉTODO}

\section{Participantes}

75 crianças e os seus pais participaram no estudo. As crianças tinham idades compreendidas entre os 38,3 e os 76,4 meses ( $M=55,47, D P=9,51)$, sendo 33 do sexo feminino e 42 do sexo masculino; e das quais 55 tinham irmãos. As crianças passavam em média 8,26 $(D P=1,08)$ horas por dia na escola. Os pais tinham idades compreendidas entre os 28 e os 50 anos $(M=39,13, D P=4,51)$, e as suas habilitações literárias variavam entre os 9 e os 21 anos de escolaridade $(M=14,57, D P=3,43)$, em que 6,8\% tinham o Ensino Básico, 45,9\% o Ensino Secundário e 47,3\% o Ensino Superior. Todos os pais trabalhavam, à exceção 
de 4. As mães tinham idades compreendidas entre os 27 e os 48 anos $(M=37,00, D P=4,04)$, e as suas habilitações literárias variavam entre os 9 e os 21 anos de escolaridade $(M=16,39, D P=2,45)$, sendo que 1,3\% tinham o Ensino Básico, 16,0\% o Ensino Secundário e 82,7\% o Ensino Superior. Todas as mães trabalhavam, à exceção de 5. Relativamente ao estado civil dos pais, apenas 14,9\% estavam separados ou divorciados, sendo que os restantes se encontravam casados ou em união de facto. As famílias foram recrutadas a partir de escolas privadas com fins lucrativos e sem fins lucrativos - IPSS dos distritos de Lisboa e Setúbal.

\section{Instrumentos}

0 Parenting Styles and Dimensions Questionnaire (PSDQ; Pedro, Carapito, \& Ribeiro, 2015; Robinson, Mandleco, Olsen, \& Hart, 2001) analisa a perceção que o pai (ou a mãe) tem acerca dos seus estilos e práticas parentais. É constituído por 32 itens organizados nas três dimensões clássicas propostas por Baumrind (1966): 1) Estilo Autoritário (12 itens), remete para o baixo suporte e elevada punição como forma de controlo (e.g., "Castigo fisicamente o meu filho para o disciplinar"); 2) Estilo Autoritativo (15 itens), remete para a responsividade às necessidades da criança e definição de limites (e.g., "Sou sensível às necessidades e sentimentos do meu filho"); e 3) Estilo Permissivo (5 itens), remete para uma elevada responsividade e baixa imposição de regras e limites (e.g., "Cedo quando o meu filho faz birra"). Os pais respondem numa escala de tipo Likert de 5 pontos (0 Nunca - 5 Sempre). Valores mais elevados numa das dimensões são indicadores de que esse estilo parental é o mais característico. Os alfas de Cronbach foram de 0,88 para o Estilo Autoritário; 0,82 para o Estilo Autoritativo; e 0,69 para o Estilo Permissivo, sendo semelhantes aos reportados por Pedro e colaboradores (2015).

O Coping with Children's Negative Emotions Scale (CCNES, Fabes, Eisenberg, \& Bernzweig, 1990) analisa a perceção que os pais têm do modo como respondem às emoções negativas dos seus filhos. Foi utilizada a versão reduzida, traduzida por Melo (2005), que é constituída por oito situações hipotéticas relacionadas com cenários do dia a dia. Nesta versão, foram retiradas situações demasiado específicas e as situações que desencadeavam emoções semelhantes a outros cenários, com o objetivo de diminuir a resistência dos pais ao preenchimento do questionário. São analisadas: 1) as Reações Centradas no Problema, que remetem para situações em os pais ajudam a criança a lidar com as emoções negativas, procurando resolver o problema que as causou; 2) as Reações Centradas nas Emoções, que se referem às situações em que os pais ajudam a criança a encontrar estratégias de regulação emocional; 3) as Reações de Encorajamento Expressivo, que se focam nas situações em que os pais aceitam e encorajam a expressão emocional negativa da criança; 4) as Reações de Minimização, que indicam as situações em que os pais desvalorizam o problema da criança e a sua expressão emocional negativa; 5) as Reações de Punição, relacionadas com a punição física ou verbal da criança aquando a sua expressão emocional negativa, de modo a diminuir a exposição ou a necessidade dos pais em lidar com a situação; e 6) as Reações de Perturbação, que indicam as situações em que o comportamento dos pais é marcado pelo desconforto e stress derivado da sua exposição às expressões emocionais negativas da criança. Cada situação hipotética apresentada inclui seis itens (cada um correspondente a um tipo de reação), sendo pedido ao pai que indique a probabilidade de responder de determinada forma numa escala de Likert de 7 pontos (1 Muito pouco provável - 7 Muito provável). É calculada a média das pontuações dos itens para cada subescala, sendo a subescala com maior valor a que traduz o tipo de reação mais frequente dos pais à expressão emocional negativa da criança. Os valores para os alfas de Cronbach foram de 0,68 para as Reações Centradas no Problema; 0,76 para as Reações Centradas na Emoção; 0,86 para as Reações de Encorajamento Expressivo; 0,83 para as Reações de Minimização; e 0,81 para as Reações de Punição. Na escala Reações de Perturbação retirou-se o item: "Se a minha criança está prestes a aparecer num papel numa festa da escola, ou nalguma atividade e fica claramente nervosa acerca do facto de as pessoas irem olhar para ela, fico calmo para não ficar eu nervoso", atingindo, o alfa, dessa forma, um valor de 0,66. Estes valores são superiores aos reportados por Melo (2005), que variam entre 0,60 e 0,75. Estes oito cenários podem ser, ainda, organizados em duas dimensões de Práticas Positivas e Práticas Negativas (Eisenberg, Fabes, \& Murphy, 1996).

0 Test of Emotion Comprehension (TEC; Dâmaso, Arriaga, \& Alexandre, 2011; Pons et al., 2004) avalia a compreensão das emoções de crianças entre os 3 e 11 anos, considerando nove dimensões: 1) o Reconhecimento da emoção com base na expressão facial apresentada; 2) a Causalidade Externa, que avalia a compreensão das causas externas das emoções; 3) a Desejabilidade, que analisa a capacidade da criança em compreender que uma mesma situação pode gerar emoções distintas em pessoas diferentes; 4) a Crença, onde a criança deve atribuir uma emoção ao protagonista que detém uma falsa crença; 5) as Lembranças, que avaliam o efeito das recordações na compreensão da emoção; 6) a Regulação foca-se na capacidade da criança em regular as suas emoções; 7) a Ocultação avalia a capacidade da criança em compreender que a expressão de um estado emocional pode não corresponder à emoção que é realmente 
sentida; 8) as Emoções Mistas, que remetem para a capacidade da criança em compreender que uma pessoa pode sentir várias emoções perante uma mesma situação; e, finalmente, 9) a Moralidade, que analisa a compreensão da emoção que resulta da prática de uma ação moralmente repreensível. Estas dimensões permitem o cálculo de um valor Global da Compreensão das Emoções. Existe uma versão dos materiais para rapazes e outra para raparigas, diferindo apenas no sexo/nome do protagonista. A cotação do TEC varia entre 0 e 9 pontos. Para esta amostra o alfa de Cronbach é de 0,68, valor semelhante ao de outros estudos (De Stasio, Fiorilli, \& Chiacchio, 2014; Ornaghi et al., 2015). Em estudos portugueses (Santos, 2012; Santos \& Franco, 2012) verificou-se que este instrumento também apresenta boas propriedades psicométricas $(\mathrm{KR}-20=.66)$.

\section{Procedimento}

Este estudo insere-se num projeto mais vasto cujo objetivo é analisar o papel do pai e do seu envolvimento no contexto familiar, no desenvolvimento socioemocional da criança, tendo seguido todas as indicações e procedimento éticos da APA e da Ordem dos Psicólogos Portugueses. Após a apresentação do projeto aos Diretores das escolas e obtenção da respetiva autorização para recolha de dados, foram enviados os consentimentos informados aos pais. Esta é uma amostra de conveniência, pelo que todas as famílias com crianças entre os 3 e 6 anos a frequentar o ensino pré-escolar, sem atrasos no desenvolvimento identificados, foram incluídas no estudo. Cerca de $60 \%$ das famílias contactadas aceitaram participar no projeto, apenas para estas foram enviados, em envelope (a ser devolvido fechado), os questionários a preencher pelo pai (CCNES e PSDQ) e foi aplicado o TEC às crianças. 0 TEC foi administrado individualmente (por um assistente de investigação previamente treinado), num espaço tranquilo disponibilizado pelas escolas, tendo uma duração aproximada de 15 minutos. Saliente-se que, antes do início da aplicação do mesmo, foi perguntado às crianças se queriam realizar esta atividade. Apenas após o seu consentimento verbal se deu início à prova. Este instrumento é constituído por dois cadernos (um para as raparigas e outro para os rapazes) que incluem um conjunto de vinhetas, sendo apresentado um cenário e lida uma história acerca do protagonista, cuja face foi propositadamente deixada em branco (e.g., "Este menino está a ser perseguido por um monstro. Como é que ele se está a sentir?"). As diversas situações devem ser descritas de forma emocionalmente neutra, eliminando pistas verbais e nãoverbais e evitando influenciar a resposta da criança. Após ouvir a história é pedido à criança que faça uma atribuição emocional ao protagonista através de uma resposta não-verbal, i.e., deve apontar para uma das quatro faces que lhe são apresentadas, sendo que as alternativas variam entre quatro emoções básicas ("feliz", "triste", "assustado" e "zangado") e uma expressão neutra ("bem-disposto"). Em alguns cenários são introduzidas perguntas de controlo, para verificar se a criança compreende os pormenores da história, sendo corrigida quando fornece uma resposta errada.

\section{Plano de análise de dados}

As análises estatísticas foram realizadas recorrendo ao SPSS Statistics (v. 21, IBM SPSS, Chicago, IL). Começou-se por analisar as medidas descritivas para os estilos parentais e práticas de socialização das emoções negativas. Foram efetuadas ANOVAS de medições repetidas no sentido de verificar a existência de diferenças entre as médias dos três estilos parentais e testes $t$ para amostras emparelhadas para testar a existência de diferenças de médias entre as práticas de socialização. De seguida, realizaram-se testes $t$ para amostras independentes no sentido de testar diferenças em função do sexo das crianças para os estilos parentais e práticas de socialização em função do sexo. Posteriormente, analisaram-se as medidas descritivas da compreensão emocional, e procedeu-se ao cálculo do teste $t$ para amostras independentes para testar diferenças em função do sexo. Em seguida, foram realizadas correlações de Pearson entre as variáveis sociodemográficas da criança, pai e mãe, os estilos parentais, as práticas de socialização e a compreensão das emoções da criança, no sentido de testar as inter-relações entre as mesmas.

Por fim, procedeu-se à realização de dois modelos de regressão hierárquica tendo a compreensão emocional como variável dependente. No primeiro modelo entraram como preditores as variáveis independentes idade e sexo da criança (bloco 1), estilos parentais autoritativo, autoritário e permissivo (bloco 2) e as práticas de socialização positivas, nomeadamente, reações centradas na emoção, no problema e de encorajamento expressivo (bloco 3). No segundo modelo mantiveram-se todas as variáveis, à exceção do bloco 3, que foi substituído pelas práticas de socialização negativas, especificamente, reações de minimização, punição e perturbação. Considerando o tamanho da amostra em estudo, recorreu-se ao método de reamostragem Bootstrap com base em 1000 amostras, já que este tipo de métodos não exige que as distribuições sejam normais, nem que as amostras sejam grandes, permitindo fazer inferência e chegar a respostas mais precisas do que os métodos tradicionais nestas circunstâncias (Moore, McCabe, Duckworth, \& Sclove, 2006). 


\section{RESULTADOS}

As medidas descritivas dos estilos parentais e práticas de socialização das emoções negativas do pai são apresentadas na Tabela 1. Refira-se que para os Estilos Parentais os valores teóricos variam entre 1 e 5, e para as Práticas de Socialização entre 1 e 7.

Tabela 1. Medidas Descritivas dos Estilos Parentais e Práticas Paternas de Socialização das Emoções Negativas

\begin{tabular}{llcccc}
\hline & & Mínimo & Máximo & $M$ & $D P$ \\
\hline Estilos Parentais & Autoritativo & 2.72 & 4.76 & 3.88 & 0.43 \\
& Autoritário & 1.00 & 3.40 & 1.71 & 0.50 \\
& Permissivo & 1.00 & 3.75 & 2.08 & 0.66 \\
\hline Práticas de & Práticas Positivas & 2.25 & 6.50 & 5.03 & 0.89 \\
Socialização & Problema & 2.13 & 7.00 & 5.24 & 0.85 \\
das Emoções & Emoções & 3.13 & 7.00 & 5.42 & 0.89 \\
Negativas & Encorajamento Expressivo & 1.13 & 6.63 & 4.44 & 1.30 \\
& Práticas Negativas & 1.00 & 5.13 & 2.58 & 0.90 \\
& Minimização & 1.00 & 5.50 & 2.90 & 1.15 \\
& Punição & 1.00 & 5.00 & 2.34 & 0.97 \\
& Perturbação & 1.00 & 5.29 & 2.48 & 0.88 \\
\hline
\end{tabular}

Os pais caracterizam-se, essencialmente, como sendo autoritativos, com 98,6\% a situarem-se acima do ponto médio da escala, e a recorrerem maioritariamente a práticas de socialização positivas, encontrando-se $88 \%$ dos mesmos acima do ponto médio da escala.

Uma ANOVA de medições repetidas permitiu verificar que existem diferenças entre as médias dos estilos parentais $\left(F_{(2,72)}=337,657 ; p<0,001 ; \eta^{2}=0,904\right.$; Potência=1,000). Com testes de comparações múltiplas (Bonferroni) a indicarem que estas diferenças são significativas entre todos os estilos parentais: o autoritário e o permissivo $(p<0,001)$, o autoritário e o autoritativo $(p<0,001)$ e o permissivo e o autoritativo $(p<0,001)$.

0 teste $t$ para amostras emparelhadas revelou uma diferença significativa entre as médias de utilização das práticas de socialização positivas e negativas $\left(t_{(74)}=17,110 ; p<0,001\right)$, verificando-se que os pais recorrem mais frequentemente às práticas de socialização positivas.

Testes $t$ para amostras independentes indicam que não existem diferenças em função do sexo da criança para os estilos parentais (autoritativo, $t_{(72)}=-0,09, p=0,93$; autoritário, $t_{(72)}=-1,31, p=0,19$; permissivo, $t_{(72)}=-1,00, p=0,32$ ), práticas de socialização positivas, $t_{(73)}=0,02, p=0,99$ (Reações Centradas no Problema, $t_{(73)}=-0,42, p=0,68$; Reações Centradas na Emoção, $t_{(73)}=-0,39, p=0,70$; Reações de Encorajamento Expressivo, $\left.t_{(73)}=0,56, p=0,58\right)$, e práticas de socialização negativas, $t_{(73)}=-1,19, p=0,24$ (Reações de Minimização, $t_{(73)}=-1,31, p=0,19$; Reações de Punição, $\left.t_{(73}\right)=-0,20, p=0,84$; Reações de Perturbação, $\left.t_{(73)}=-1,89, p=0,06\right)$.

Em relação à Compreensão Emocional das crianças, o valor teórico para a Compreensão Global varia entre 0 e 9. As crianças acertaram no mínimo em 0 componentes e no máximo em 8 , sendo a média de respostas corretas de 3,96 $(D P=1,72)$. 0 teste $t$ para amostras independes indicou que não existem diferenças em função do sexo $\left(t_{(73)}=1,69, p=0,096\right)$.

Na Tabela 2 são apresentadas as correlações de Pearson entre as variáveis sociodemográficas da criança, do pai e da mãe, os estilos parentais, as práticas de socialização e a compreensão das emoções da criança, no sentido de testar as inter-relações entre as mesmas. A idade do pai está positiva e significativamente correlacionada com o estilo autoritativo; e as habilitações literárias do pai com o estilo autoritário, com a dimensão práticas de socialização negativas, assim como, com as práticas de minimização, punição e perturbação. A idade da mãe encontra-se positiva e significativamente correlacionada com as práticas de socialização positivas e com as práticas centradas na emoção. As habilitações literárias da mãe estão positiva e significativamente correlacionadas com as práticas de socialização negativas, de minimização, punição e perturbação. 0 número de horas de trabalho semanal da mãe está negativa e significativamente associado ao estilo autoritativo do pai, às suas práticas de socialização positivas, e às práticas centradas no problema e centradas na emoção.

O estilo parental autoritativo está positiva e significativamente associado com a dimensão práticas de socialização positivas e com as práticas centradas no problema, na emoção e de encorajamento expressivo. Os estilos parentais autoritário e permissivo encontram-se positiva e significativamente correlacionados com a dimensão práticas de socialização negativas e com as práticas de minimização, punição e perturbação. 
Tabela 2. Correlações entre as Variáveis Sociodemográficas, Estilos Parentais e Práticas Paternas de Socialização das Emoções Negativas, Compreensão Emocional da criança

\begin{tabular}{|c|c|c|c|c|c|c|c|c|c|c|c|c|c|c|c|c|c|c|c|}
\hline & 1 & 2 & 3 & 4 & 5 & 6 & 7 & 8 & 9 & 10 & 11 & 12 & 13 & 14 & 15 & 16 & 17 & 18 & 19 \\
\hline 1. Idade Criança & - & -0.04 & 0.17 & -0.16 & -0.17 & -0.19 & -0.17 & 0.13 & -0.12 & -0.15 & 0.09 & 0.12 & 0.03 & 0.08 & -0.09 & -0.17 & 0 & -0.07 & $0.54^{* *}$ \\
\hline 2. Idade Mãe & - & - & $0.54^{* *}$ & 0.21 & -0 & 0.02 & 0.09 & 0.21 & 0.07 & -0 & $0.25^{*}$ & 0.17 & $0.28^{*}$ & 0.21 & 0 & 0.11 & -0.1 & -0.1 & 0.14 \\
\hline 3. Idade Pai & - & - & - & 0.01 & 0.11 & 0.03 & -0.1 & $0.23^{*}$ & 0.09 & -0.1 & 0.09 & 0.09 & 0.09 & 0.08 & -0 & 0 & 0.05 & -0.1 & 0 \\
\hline 4. Hab. Lit. Mãe & - & - & - & - & $0.52^{* *}$ & -0.08 & $0.26^{*}$ & -0.12 & 0.23 & 0.13 & -0.08 & -0.08 & -0.12 & -0.04 & $0.39^{* *}$ & $0.44^{* *}$ & $0.28^{*}$ & $0.30^{* *}$ & -0.07 \\
\hline 5. Hab. Lit. Pai & - & - & - & - & - & -0.15 & 0.03 & 0 & $0.28^{*}$ & 0.15 & -0.03 & -0.01 & -0.1 & 0 & $0.42^{* *}$ & $0.39^{* *}$ & $0.40^{* *}$ & $0.34^{*}$ & -0.13 \\
\hline 6. Horas de Trab/Mãe & & & & & & & 0.05 & $-0.30 *$ & 0.06 & 0.13 & $-0.31^{*}$ & $-0.33^{* *}$ & $-0.30 *$ & -0.21 & 0.02 & 0.04 & -0.02 & 0.03 & -0.16 \\
\hline 7. Horas de Trab/Pai & - & - & - & - & - & - & - & -0.02 & -0.04 & -0.06 & 0.08 & 0.1 & 0.15 & -0.01 & -0.03 & 0.03 & -0.1 & -0.03 & 0.16 \\
\hline 8. Estilo Autoritativo & - & - & - & - & - & - & - & - & -0.2 & $-0.23 *$ & $0.48^{* *}$ & $0.46^{* *}$ & $0.47^{* *}$ & $0.37^{* *}$ & -0.16 & -0.09 & -0.15 & -0.22 & -0.03 \\
\hline 9. Estilo Autoritário & - & - & - & - & - & - & - & - & - & $0.57^{* *}$ & -0.2 & -0.2 & -0.11 & -0.19 & $0.58^{* *}$ & $0.48^{* *}$ & $0.58^{* *}$ & $0.50^{* *}$ & -0.12 \\
\hline 10. Estilo Permissivo & - & - & - & - & - & - & - & - & - & - & -0.12 & -0.12 & -0.11 & -0.08 & $0.43^{* *}$ & $0.37^{* *}$ & $0.33^{* *}$ & $0.48^{* *}$ & $-0.27^{*}$ \\
\hline 11. Práticas Positivas & - & - & - & - & - & - & - & - & - & - & - & $0.90^{* *}$ & $0.84^{* *}$ & $0.89^{* *}$ & 0.04 & 0.12 & -0.04 & -0.01 & 0.04 \\
\hline 12. Problema & - & - & - & - & - & - & - & - & - & - & - & - & $0.75^{* *}$ & $0.67^{* *}$ & 0.01 & 0.07 & -0.1 & 0.02 & 0.03 \\
\hline 13. Emoção & - & - & - & - & - & - & - & - & - & - & - & - & - & $0.55^{* *}$ & 0 & 0.12 & -0.1 & -0.1 & 0.1 \\
\hline 14. Encoraj. Express. & - & - & - & - & - & - & - & - & - & - & - & - & - & - & 0.07 & 0.13 & 0 & 0.05 & -0.01 \\
\hline 15. Práticas Negativas & - & - & - & - & - & - & - & - & - & - & - & - & - & - & - & $0.92^{* *}$ & $0.91^{* *}$ & $0.87^{* *}$ & -0.21 \\
\hline 16. Minimiz. & - & - & - & - & - & - & - & - & - & - & - & - & - & - & - & - & $0.73^{* *}$ & $0.69^{* *}$ & -0.2 \\
\hline 17. Punição & - & - & - & - & - & - & - & - & - & - & - & - & - & - & - & - & - & $0.71^{* *}$ & -0.1 \\
\hline 18. Perturb. & - & - & - & - & - & - & - & - & - & - & - & - & - & - & - & - & - & - & $-0.28^{*}$ \\
\hline 19. Comp. Emocional & - & - & - & - & - & - & - & - & - & - & - & - & - & - & - & - & - & - & - \\
\hline
\end{tabular}

Nota: ${ }^{*} p \leq 0.05 .{ }^{* *} p<0.001$ 
A idade das crianças encontra-se positiva e significativamente associada com a sua compreensão emocional. Esta está negativa e significativamente associada com as reações de perturbação e com o estilo parental permissivo do pai.

De seguida testaram-se dois modelos de regressão hierárquica, tendo a compreensão emocional da criança como variável dependente. No primeiro modelo entraram como preditores as variáveis independentes idade e sexo da criança (bloco 1), os estilos parentais autoritativo, autoritário e permissivo (bloco 2), e as práticas de socialização positivas, nomeadamente, reações centradas na emoção, no problema e de encorajamento expressivo (bloco 3). No segundo modelo mantiveram-se todas as variáveis, à exceção do bloco 3 , que foi substituído pelas práticas de socialização negativas, especificamente, reações de minimização, punição e perturbação. Os valores VIF apresentaram-se todos abaixo de 5, o que indica não existirem problemas de multicolinearidade. Em ambos os modelos verificamos que a idade da criança é a única variável que permite predizer significativamente a compreensão emocional. Nas Tabelas 3 e 4 são apresentados os valores da regressão múltipla hierárquica para os dois modelos.

Tabela 3. Regressão Múltipla Hierárquica para o Modelo 1 - Idade e Sexo da Criança, Estilos Parentais e Práticas de Socialização Positivas como Preditores

\begin{tabular}{|c|c|c|c|c|c|}
\hline \multirow{2}{*}{ Bloc } & & \multicolumn{4}{|c|}{ Compreensão Emocional } \\
\hline & & $\mathrm{B}$ & SE B & $\beta$ & $\Delta \mathrm{R}^{2}$ \\
\hline \multirow[t]{2}{*}{1} & Sexo & -0.564 & 0.348 & -0.162 & $0.318^{* * *}$ \\
\hline & Idade & $0.097^{* *}$ & 0.018 & 0.532 & \\
\hline \multirow[t]{5}{*}{2} & Sexo & -0.490 & 0.352 & -0.141 & 0.053 \\
\hline & Idade & $0.095^{* *}$ & 0.018 & 0.523 & \\
\hline & Estilo Autoritativo & -0.538 & 0.404 & -0.132 & \\
\hline & Estilo Autoritário & 0.283 & 0.428 & 0.082 & \\
\hline & Estilo Permissivo & -0.670 & 0.365 & -0.251 & \\
\hline \multirow[t]{10}{*}{3} & Sexo & -0.543 & 0.353 & -0.156 & 0.039 \\
\hline & Idade & $0.099 * *$ & 0.017 & 0.544 & \\
\hline & Estilo Autoritativo & -0.764 & 0.460 & -0.187 & \\
\hline & Estilo Autoritário & 0.199 & 0.439 & 0.058 & \\
\hline & Estilo Permissivo & -0.616 & 0.355 & -0.231 & \\
\hline & Reações Centradas no Problema & -0.296 & 0.343 & -0.146 & \\
\hline & Reações Centradas na Emoção & 0.597 & 0.312 & 0.306 & \\
\hline & Encorajamento Expressivo & -0.094 & 0.177 & -0.071 & \\
\hline & $\mathrm{R}^{2}$ & \multicolumn{4}{|c|}{$0.409^{* * *}$} \\
\hline & $\mathrm{R}^{2}$ ajustado & \multicolumn{4}{|c|}{$0.335^{* * *}$} \\
\hline
\end{tabular}

Nota: ${ }^{*} \mathrm{p}<0,05 ;{ }^{* *} \mathrm{p}<0,01 ;{ }^{* * *} \mathrm{p}<0,001$ (Regressão hierárquica com estimação bootstrap. Standard errors calculados com base em 1000 amostras bootstrap.)

Tabela 4. Regressão Múltipla Hierárquica para o Modelo 2 - Idade e Sexo da Criança, Estilos Parentais e Práticas de Socialização Negativas como Preditores

\begin{tabular}{|c|c|c|c|c|c|}
\hline \multirow{2}{*}{ Bloc } & & \multicolumn{4}{|c|}{ Compreensão Emocional } \\
\hline & & B & SE B & $\beta$ & $\Delta R^{2}$ \\
\hline \multirow[t]{2}{*}{1} & Sexo & -0.564 & 0.359 & -0.162 & $0.318^{* * *}$ \\
\hline & Idade & $0.097^{* *}$ & 0.018 & 0.532 & \\
\hline \multirow[t]{5}{*}{2} & Sexo & -0.490 & 0.349 & -0.141 & 0.053 \\
\hline & Idade & $0.095^{* *}$ & 0.017 & 0.523 & \\
\hline & Estilo Autoritativo & -0.538 & 0.415 & -0.132 & \\
\hline & Estilo Autoritário & 0.283 & 0.432 & 0.082 & \\
\hline & Estilo Permissivo & -0.670 & 0.380 & -0.251 & \\
\hline \multirow[t]{10}{*}{3} & Sexo & -0.471 & 0.347 & -0.135 & 0.054 \\
\hline & Idade & $0.102^{* *}$ & 0.019 & 0.564 & \\
\hline & Estilo Autoritativo & -0.705 & 0.439 & -0.173 & \\
\hline & Estilo Autoritário & 0.608 & 0.463 & 0.176 & \\
\hline & Estilo Permissivo & -0.554 & 0.399 & -0.207 & \\
\hline & Minimização & 0.311 & 0.230 & 0.202 & \\
\hline & Punição & -0.283 & 0.326 & -0.155 & \\
\hline & Perturbação & -0.529 & 0.345 & -0.260 & \\
\hline & & \multicolumn{4}{|c|}{$0,424^{* * *}$} \\
\hline & & \multicolumn{4}{|c|}{$0,352^{* * *}$} \\
\hline
\end{tabular}

Nota: ${ }^{*} \mathrm{p}<0,05 ;{ }^{* *} \mathrm{p}<0,01 ;{ }^{* * *} \mathrm{p}<0,001$ (Regressão hierárquica com estimação bootstrap. Standard errors calculados com base em 1000 amostras bootstrap.) 


\section{DISCUSSÃO}

Nos últimos anos têm-se assistido a diversas transformações sociais, económicas e políticas com implicações profundas nas vivências e organização das famílias, assim como no modo como a parentalidade é vivenciada por ambas as figuras cuidadoras, promovendo um maior envolvimento do pai (TamisLeMonda \& Cabrera, 2002). A evolução do papel do pai como guia moral, para um pai cuidador e afetuoso evidencia a sua capacidade de dar resposta adequada às necessidades físicas e emocionais da criança (ver Lamb, 2010). No entanto, a maioria da investigação centra-se, ainda, na análise das variáveis maternas preditoras do desenvolvimento socioemocional da criança. Dada esta lacuna, e considerando que a capacidade de compreender emoções na infância assenta, entre outros, em processos de socialização (Denham, 1998), é expectável que o pai tenha um papel de relevância no desenvolvimento desta competência (Havighurst et al., 2010), pelo que o presente estudo procurou analisar as inter-relações entre os estilos parentais paternos, as práticas de socialização paternas das emoções negativas e a compreensão das emoções de crianças em idade pré-escolar.

Os pais que participaram no estudo apresentam, essencialmente, um estilo autoritativo (e.g., Monteiro, Fernandes, Torres, \& Santos, 2017; Pedro et al., 2015) e recorrem mais frequentemente a práticas de socialização positivas, tal como descrito na literatura mais recente (e.g., Mazzone \& NaderGrosbois, 2016). Estes resultados vão no sentido da mudança de paradigma, no qual encontramos um pai menos autoritário e disciplinador, mais afetuoso e participativo nas rotinas e cuidados diários da criança (ver Lamb, 2010).

Verificou-se que a idade do pai está positivamente associada com o estilo autoritativo. De acordo com alguns estudos (e.g., Halpenny et al., 2010), pais com idades mais elevadas permitem que os filhos participem nas decisões familiares e procuram evitar o uso de punição física e ameaças; parecendo sentirse mais à vontade para gerir as situações mais difíceis e exigentes da parentalidade (Dietz, 2000). Seria expectável que pais com um nível de escolaridade mais elevado demonstrassem mais conhecimento acerca das necessidades da criança e das estratégias mais eficazes relativas à parentalidade (Monteiro et al., 2017; Morawska, Winter, \& Sanders, 2009), potencializando deste modo a utilização de práticas de socialização mais positivas e do estilo autoritativo. No entanto, nesta amostra encontrou-se uma associação positiva e significativa entre o estilo autoritário e as habilitações literárias do pai, que poderá ser consequência do aumento do seu envolvimento nas tarefas familiares e da necessidade de conciliação com as responsabilidades profissionais, i.e., com a exigência e encargos que trabalhos mais especializados possam ter. Além disso, as experiências laborais poderão constituir-se como fonte de stress e possível causa para adoção deste estilo (Niez \& Alico, 2015; Slep \& 0'Leary, 2007).

Verificou-se, também, a existência de uma relação positiva e significativa entre as habilitações literárias da mãe e as práticas de socialização negativas do pai; e de uma relação negativa e significativa entre o número de horas de trabalho da mãe e o estilo autoritativo e as práticas de socialização positivas do pai (e.g., Monteiro et al., 2017). Poder-se-á colocar como hipótese explicativa que nas famílias de duploemprego, onde as mães poderão assumir cargos mais especializados, os pais tenham de assumir maiores responsabilidades nos cuidados à criança (e.g., Pimenta, Veríssimo, Monteiro, \& Pessoa e Costa, 2010). Estas circunstâncias poderão gerar dificuldades e stressores no equilíbrio da vida doméstica e profissional (Belsky, 1984), traduzindo-se na menor utilização de práticas de socialização positivas. Contudo, esta hipótese explicativa deverá ser clarificada em estudos futuros.

Tal como noutros estudos (e.g., Gottman \& DeClaire, 1997; Topham et al., 2011), verificou-se que, nesta amostra, pais com um estilo autoritativo tendem a utilizar práticas de socialização das emoções mais positivas, enquanto os estilos autoritário e permissivo estão associados com o uso de práticas de socialização emocional mais negativas. Os cuidadores com um estilo autoritativo tendem a ser responsivos às necessidades da criança e a considerar os seus interesses individuais e pontos de vista quando estas se opõem às regras dos pais (Baumrind, 1966). Neste estilo espera-se que exista aceitação e suporte às emoções e compreensão das emoções da criança, associados com as práticas de socialização positivas (Fabes et al., 2002). Contrariamente, os estilos autoritário e permissivo encontram-se associados a práticas de socialização negativas caracterizadas pela punição, desvalorização das emoções da criança e desconforto face às mesmas (Topham et al., 2011).

De acordo com a literatura centrada na figura materna (e.g., Bennet et al., 2005; Fabes et al., 2002), bem como nos estudos que incluem ambos os pais como participantes (e.g., Denham et al., 1997; Gottman \& DeClaire, 1997), esperava-se que o estilo autoritativo e as práticas de socialização positivas estivessem associadas a níveis mais elevados de compreensão emocional, e o inverso se verificasse com os estilos autoritário e permissivo e as práticas tidas como mais negativas. No presente estudo verificou-se que apenas o estilo parental permissivo e as reações de perturbação do pai apresentam uma associação negativa com a compreensão emocional, tal como expectável. A permissividade implica uma baixa imposição de regras, falta de orientação do comportamento da criança, a não exposição ao confronto com 
a mesma e a crença de que as emoções da criança são irracionais (Baumrind, 1966; Niez \& Alico, 2015), enquanto as reações de perturbação remetem para o desconforto parental face à emoção negativa da criança (Fabes et al., 2002). Neste sentido, implicam uma incapacidade de transmitir à criança as informações necessárias para a interpretação das situações que envolvem a emoção, prejudicando a sua compreensão emocional (Denham, 1998; Havighurst et al., 2010; Sullivan et al., 2010).

A maioria da investigação, realizada com mães, tende a indicar uma relação negativa entre estilo autoritário e práticas negativas com a compreensão emocional da criança em idade pré-escolar. No entanto, Guajardo e colaboradores (2009) não encontraram relação entre as demonstrações de raiva ou irritabilidade de ambos os pais e a compreensão emocional, sugerindo que estes comportamentos poderão afetar o desenvolvimento cognitivo da criança, mas não especificamente a compreensão das emoções. Também, para o estilo autoritativo e práticas de socialização positivas do pai não se encontraram associações com a compreensão emocional, indo ao encontro dos resultados obtidos por Niez e Alico (2015). Estes não encontraram associações entre os estilos parentais dos cuidadores (mãe/pai) e o desenvolvimento socioemocional da criança (que inclui a compreensão emocional), considerando que características como o temperamento da criança possam ter um maior impacto nestas competências.

Tal como expectável, verificou-se que quanto mais elevada a idade das crianças, mais elevados os valores de compreensão emocional no TEC (Karstad, Wishstrøm, Reinfjell, Belsky, \& Berg-Nielsen, 2015; Pons et al., 2004; Sagone \& Caroli, 2014). A compreensão emocional é constituída por um conjunto de competências que se desenvolvem, não só através da experiência emocional ao longo do tempo e em diferentes contextos, mas também com o desenvolvimento da linguagem (Karstad et al., 2015), a integração e o processamento da informação (Albanese, De Stasio, Chiacchio, Fiorilli, \& Pons, 2010). Assim, já nos anos pré-escolares as crianças com idades superiores apresentam uma boa capacidade de identificar as expressões emocionais e as causas das emoções (Pons et al., 2004).

As interpretações destes resultados merecem, contudo, cautela dadas as limitações do estudo relativamente ao número de participantes e à pouca diversidade da amostra em termos sociodemográficos. Refira-se, ainda, que a maioria dos pais se perceciona como tendo um estilo autoritativo e recorrendo a práticas de socialização positivas tornando esta uma amostra homogénia relativamente aos preditores da compreensão emocional.

Em estudos futuros será importante analisar outras variáveis da criança, das figuras parentais, bem como outros parceiros de interação significativos. Por exemplo, será aconselhável controlar a competência verbal da criança, dado que na literatura são reportadas associações positivas com a sua compreensão emocional (e.g., De Stasio et all., 2014; Martins, Osório, Veríssimo, \& Martins, 2016; Morgan, Izard, \& King, 2010). Embora o objetivo do estudo fosse analisar as contribuições do Pai para a compreensão emocional da criança, há que considerar, em simultâneo, os contributos de estilos parentais e práticas de socialização da mãe, de modo a compreender os efeitos independentes e de interação de ambos. Considerando as idades das crianças, outras dimensões da parentalidade deverão ser analisadas, como por exemplo, a sensibilidade dos comportamentos parentais (Ainsworth, 1999). Deverá ser, igualmente, explorado se pais mais envolvidos nos cuidados à criança e em atividades de brincadeira com a mesma poderão ter maior impacto ao nível da compreensão emocional (Monteiro et. al., 2010; Parke et. al., 2006). Para além das figuras cuidadoras, no sistema familiar, os irmãos têm o potencial de desempenhar um importante papel no desenvolvimento socioemocional, pelo que deverão ser considerados nas análises (e.g., Denham, 1998; Eisenberg et al., 1998).

Os anos pré-escolares são marcados pela tarefa de entrada, estabelecimento e manutenção de interações positivas da criança com o grupo de pares (Belacchi \& Farina, 2010; Parke et al., 2006; ZahnWaxler, 2010). Este contexto potencializa o desenvolvimento da capacidade de identificação, interpretação e compreensão correta das emoções e comportamentos dos outros, de modo a agir em concordância com as normas de expressão emocional implícitas do grupo e ser aceite pelo mesmo, tornando-se por isso um fator a considerar na análise do desenvolvimento da compreensão emocional (Denham et al., 2003).

Apesar das limitações identificadas, este estudo visou contribuir para o aumento do conhecimento sobre o papel do pai, dado que apesar do crescente interesse por parte da comunidade científica e do seu maior envolvimento na vida da criança, continua a ser uma figura menos explorada, comparativamente com a mãe (Lamb, 2010; Wilson \& Prior, 2011). Sendo a compreensão emocional um elemento fundamental na adaptação saudável do indivíduo ao mundo que o rodeia, será relevante continuar a explorar a importância do pai, e a considerá-lo aquando o desenvolvimento de programas que promovam uma parentalidade ajustada.

\section{REFERÊNCIAS}

Ainsworth, M. D. (1999). Maternal Sensitivity Scales: The Baltimore Longitudinal Project (1969). 
Albanese, O., De Stasio, S., Chiacchio, C., Fiorilli, C., \& Pons, F. (2010). Emotion comprehension: The impact of nonverbal intelligence. The Journal of Genetic Psychology, 171 (2), 101-115.

Baumrind, D. (1966). Effects of authoritative parental control on child behavior. Child Development, 37 (4), 887-907.

Belacchi, C., \& Farina, E. (2010). Prosocial/hostile roles and emotion comprehension in preschoolers. Agressive Behavior, 36, 371-389. http://dx.doi.org/10.1002/ab.20361

Belsky, J. (1984). The determinants of parenting: A process model. Child Development, 55 (1), 83-96.

Bennet, D., Bendersky, M., \& Lewis, M. (2005). Antecedents of emotion knowledge: Predictors of individual differences in young children. Cognition and Emotion, 19 (3), 375-396. http://dx.doi.org/10.1080/02699930441000201

Bibi, F., Chaudhry, A., Awan, E., \& Tariq, B. (2013). Contribution of parenting style in life domain of children. Journal of Humanities and Social Science, 12 (2), 91-95. http://dx.doi.org/10.9790/0837-1229195

Dâmaso, P. P., Arriaga, P., \& Alexandre, J. (2011). Especificidade na diversidade: A compreensão emocional em crianças com dificuldades de aprendizagem (Dissertação de Mestrado). ISCTE-IUL, Lisboa, Portugal.

Darling, N., \& Steinberg, L. (1993). Parenting style as a context: An integrative model. Psychological Bulletin, 113, 487-496. http://dx.doi.org/10.1037/0033-2909.113.3.487

De Stasio, S., Fiorilli, C., \& Di Chiacchio, C. (2014). Effects of verbal ability and fluid intelligence on children's emotion understanding. International Journal of Psychology, 49 (5), 409-414. http://dx.doi.org/10.1002/ijop.12032

Denham, S. A. (1998). Emotional development in young children. New York: The Guilford Press.

Denham, S. A., \& Kochanoff, A. T. (2002). Parental contributions to preschoolers' understanding of emotion. Marriage \& Family Review, 34 (3-4), 311-343. http://dx.doi.org/10.1300/J002v34n03_06

Denham, S. A., Bassett, H. H., \& Wyatt, T. (2007). The socialization of emotional competence. In J. Grusec \& P. Hastings (Eds.), The handbook of socialization (pp.614-637). New York: Guilford Press.

Denham, S. A., Blair, K. A., DeMulder, E., Levitas, J., Sawyer, K., Auerbach-Major, S., \& Queenan, P. (2003). Preschool emotional competence: Pathway to social competence? Child Development, 74 (1), 238256. http://dx.doi.org/10.1111/1467- 8624.00533

Denham, S. A., Mitchell-Copeland, J., Strandberg, K., Auerbach, S., \& Blair, K. (1997). Parental contributions to preschoolers' emotional competence: Direct and indirect effects. Motivation and Emotion, 21 (1), 65-86.

Dietz, T. (2000). Disciplining children: Characteristics associated with the use of corporal punishment. Child Abuse \& Neglect, 24 (12), 1529-1542. http://dx.doi.org/10.1016/S0145-2134(00)00213-1

Eisenberg, N., Cumberland, A., \& Spinrad, T. (1998). Parental socialization of emotion. Psychological Inquiry, 9 (4), 241-273. http://dx.doi.org/10.1207/s15327965pli0904_1

Eisenberg, N., Fabes, R., \& Murphy, B. (1996). Parents' reactions to children's negative emotion: Relations to children's social competence and comforting behavior. Child Development, 67 (5), 2227-2247. http://dx.doi.org/10.2307/1131620

Fabes, R., Eisenberg, N., \& Bernzweig, J. (1990). The Coping with Children's Negative Emotions Scale: Procedures and scoring. Available from authors. Arizona State University.

Fabes, R., Poulin, R., Eisenberg, N., \& Madden-Derdich, D. (2002). The Coping with Children's Negative Emotions Scale (CCNES): Psychometric properties and relations with children's emotional competence. Marriage and Family Review, 34 (3-4), 285310.http://dx.doi.org/10.1300/J002v34n03_05

Figueiredo, A. M., Mateus, V., Osório, A., \& Martins, C. (2014). A contribuição da sensibilidade materna e paterna para o desenvolvimento cognitivo de crianças em idade pré-escolar. Análise Psicológica, 32 (2), 231-242. http://dx.doi.org/10.14417/ap.842

Friedlmeier, W., Corapci, F., \& Cole, P. (2011). Emotion socialization in cross-cultural perspective. Social and Personality Psychology Compass, 5 (7), 410-427. http://dx.doi.org/10.1111/j.17519004.2011.00362.x

Gottman, J., \& DeClaire, J. (1997). Assessing your parenting style. In J. Gottman \& J. DeClaire (Eds.), Raising an emotionally intelligent children: The heart of parenting (pp.42-68). New York: Simon and Schuster Paperbacks.

Guajardo, N., Snyder, G., \& Petersen, R. (2009). Relationships among parenting practices, parental stress, child behaviour, and children's social-cognitive development. Infant and Child Development, 18, 3760. http://dx.doi.org/10.1002/icd.578

Halberstadt, A., \& Lozada, F. (2011). Emotion development in infancy through the lens of culture. Emotion Review, 3 (2), 158-168. http://dx.doi.org/10.1177/1754073910387946 
Halpenny, A., Nixon, E., \& Watson, D. (2010). Parents' perspectives on parenting styles and disciplining children. Dublin: The Stationery Office.

Havighurst, S., Wilson, K., Harley, A., Prior, M., \& Kehoe, C. (2010). Tuning in to kids: Improving emotion socialization practices in parents of preschool children - Findings from a community trial. Journal of Child Psychology and Psychiatry, 51 (12), 1342-1350. http://dx.doi.org/10.1111/j.14697610.2010.02303.X

Jia, S., Wang, L., \& Shi, Y. (2014). Relationship between parenting and proactive versus reactive aggression among chinese preschool children. Archives of Psychiatric Nursing, 28 (2), 152-157. http://dx.doi.org/10.1016/j.apnu.2013.12.001

Karstad, S., WichstrØm, L., Reinfjell, T., Belsy, J., \& Berg-Nielsen, T. (2015). What enhances the development of emotion understanding in young children? A longitudinal study of interpersonal predictors. British Journal of Developmental Psychology, 33, 340-354. http://dx.doi.org/10.1111/bjdp.12095

Lamb, M. E. (2010). The role of the father in child development ( $5^{\text {th }}$ ed.). Hoboken, New Jersey: John Wiley \& Sons, Inc.

Leung, C., Lo, S., Tsang, S., Chan, R., \& Kung, E. (2016). The relationship between family dining practices, parenting style and family functioning and child learning. International Journal on Disability and Human Development, 15 (3), 267-276. http://dx.doi.org/10.1515/ijdhd-2015-0013

Martins, E., Osório, A., Veríssimo, M., \& Martins, C. (2016). Emotion understanding in preschool children: The role of executive functions. International Journal of Behavioral Development, 40 (1), 1-10. $10.1177 / 0165025414556096$

Mazzone, S., \& Nader-Grosbois, N. (2016). How are parental reactions to children's emotions related to their theory of mind abilities? Psychology, 7, 166-179. http://dx.doi.org/10.4236/psych.2016.72019

McElwain, N. L., Halberstadt, A. G., \& Volling, B. L. (2007). Mother-and father-reported reactions to children's negative emotions: Relations to young children's emotional understanding and friendship quality. Child Development, 78 (5), 1407-1425.

Melo, A. (2005). Emoções no período escolar: Estratégias parentais face à expressão emocional e sintomas de internalização e externalização da criança (Dissertação de Mestrado). Universidade do Minho, Braga, Portugal.

Molina, P., Bulgarelli, D., Henning, A., \& Aschersleben, G. (2014). Emotion understanding: A cross-cultural comparison between Italian and German preschoolers. European Journal of Developmental Psychology, 11 (5), 592-607. http://dx.doi.org/10.1080/17405629.2014.890585.

Monteiro, L., Fernandes, M., Torres, N., \& Santos, C. (2017). Father's involvement and parenting styles in Portuguese families: The role of education and working hours. Análise Psicológica, 4 (XXXV), 513528. http://dx.doi.org/10.14417/ap.1451

Monteiro, L., Fernandes, M., Veríssimo, M., Costa, I., Torres, N., \& Vaughn, B. (2010). Perspectiva do pai acerca do seu envolvimento em famílias nucleares. Associações com o que é desejado pela mãe e com as características da criança. Revista Interamericana de Psicología/Interamerican Journal of Psychology, 44 (1), 120-130.

Monteiro, L., Veríssimo, M., Santos, A., \& Vaughn, B. (2008). Envolvimento paterno e organização dos comportamentos de base segura das crianças em famílias portuguesas. Análise Psicológica, 26 (3), 395-409.

Moore, D. S., McCabe, G. P., Duckworth, W. M., \& Sclove, S. L. (2006). A prática da estatística empresarial: Como usar dados para tomar decisões. Rio de Janeiro: LTC Editora

Morawska, A., Winter, L., \& Sanders, M. R. (2009). Parenting knowledge and its role in the prediction of dysfunctional parenting and disruptive child behaviour. Child: Care, Health and Development, 35 (2), 217-226. http://dx.doi.org/10.1111/j.1365-2214.2008.00929.x

Morgan, J., Izard, C. E., \& King, K. (2010). Construct validity of the Emotion Matching Task: Preliminary evidence for convergent and criterion validity of a new emotion knowledge measure for young children. Social Development, 19 (1), 52-70. http://dx.doi.org/10.1111/j.1467-9507.2008.00529.x

Niez, C., \& Alico, J. (2015). Relationship of parenting styles to pre-schoolers' socio-emotional competence and academic performance. International Journal of Innovation and Research in Educational Sciences, 2 (3), 246-252.

Ornaghi, V., Grazzani, I., Cherubin, E., Conte, E., \& Piralli, F. (2015). “Let's talk about emotions!”. The effect of conversational training on preschoolers' emotion comprehension and prosocial orientation. Social Development, 24 (1), 166-183. http://dx.doi.org/10.1111/sode.12091

Parke, R., Simpkins, S., McDowell, D., Kim, M., Killian, C., Dennis, J., Flyr, M., Wild, M., \& Rah, Y. (2006). Relative contributions of families and peers to children's social development. In P. K. Smith \& C. H. Hart (Eds.), Blackwell handbook of childhood social development (pp.156-204). Oxford, UK: Blackwell Publishing. 
Pedro, M., Carapito, E., \& Ribeiro, T. (2015). Parenting Styles and Dimensions Questionnaire - Versão portuguesa de autorrelato. Psicologia: Reflexão e Crítica, 28 (2), 302-312. http://dx.doi.org/10.1590/1678-7153.201528210

Perista, H., Carsoso, A., Brázia, A., Abrantes, M., \& Perista, P. (2016). Os usos do tempo de homens e mulheres em Portugal. Policy brief. Lisboa: CESIS - Centro de Estudos para a Intervenção Social e CITE Comissão para a Igualdade no Trabalho e no Emprego.

Pimenta, M., Veríssimo, M., Monteiro, L., \& Pessoa e Costa, I. (2010). O envolvimento paterno de crianças a frequentar o jardim-de-infância. Análise Psicológica, 28 (4), 565-580. http://dx.doi.org/10.14417/ap.375

Pons, F., Harris, P. L., \& De Rosnay, M. (2004). Emotion comprehension between 3 and 11 years: Developmental periods and hierarchical organization. European Journal of Developmental Psychology, 1 (2), 127-152. http://dx.doi.org/10.1080/17405620344000022.

Reed, L. (2015). Early socialization. International Journal of Childbirth Education, 30 (2), 31-34.

Robinson, C. C., Mandleco, B., Olsen, S. F., \& Hart, C. H. (2001). The parenting styles and dimensions quastionnaire. In B. F. Perlmutter, J. Touliatos, \& G. W. Holden (Eds.), Handbook of family measurement techniques: Vol. 3. Instruments \& index (pp. 319-321). Thoausand Oaks, CA: Sage.

Rocha, A., Roazzi, A., Silva, A., Candeias, A., Minervino, C., Roazzi, M., \& Pons, F. (2013). Test of Emotional Comprehension: Exploring the underlying structure through Confirmatory Factor Analysis and Similarity Structure Analysis. In A. Roazzi, B. C. de Souza, \& W. Bilsky (Eds.), Searching fos structure in complex social, cultural and psychological phenomena (pp. 74-95). Recife, PE: FTA.

Root, A., \& Rubin, K. (2010). Gender and parents' emotion socialization beliefs during the preschool years. New Directions for Child and Adolescent Development, 128, 51-64. http://dx.doi.org/10.1002/cd.268

Sagone, E., \& De Caroli, M. (2014). Emotion comprehension and divergent thinking: What's their relationship in developmental age? Procedia - Social and Behavioral Sciences, 116, 585-589. http://dx.doi.org/10.1016/j.sbspro.2014.01.261

Santos, N. (2012). Inteligência Emocional: A compreensão das emoções em crianças do pré-escolar (Dissertação de Mestrado). Universidade da Madeira, Funchal, Portugal.

Santos, N., \& Franco, G. (2012). Inteligência emocional: A compreensão das emoções em crianças do préescolar. In L. S. Almeida, B. D. Silva, \& A. Franco (Eds.), Atas do II seminário internacional “Contributos da psicologia em contextos educativos" (pp. 1556-1567). Minho: Centro de Investigação em Educação, Instituto de Educação da Universidade do Minho.

Silva, E. (2012). A Relação entre Inteligência Emocional e o Rendimento Escolar em Crianças do 1. ․ Ciclo do Ensino Básico da R.A.M. (Dissertação de Mestrado). Universidade da Madeira, Funchal, Portugal.

Slep, A., \& O'Leary, S. (2007). Multivariate models of mothers' and fathers' aggression toward their children. Journal of Consulting and Clinical Psychology, 75 (5), 739-751. http://dx.doi.org/10.1037/0022006X.75.5.739

Sullivan, M., Carmody, D., \& Lewis, M. (2010). How neglect and punitiveness influence emotion knowledge. Child Psychiatry and Human Development, 41 (3), 285-298. http://dx.doi.org/10.1007/s10578-0090168-3

Taleb, T. (2013). Parenting styles and children's social skills as perceived by Jordanian mothers of preschool children. Early Child Development and Care, 183 (11), 1646-1660. http://dx.doi.org/10.1080/03004430.2012.744988

Tamis-LeMonda, C., \& Cabrera, N. (2002). Handbook of father involvement: Multidisciplinary perspectives. New Jersey: Lawrence Erlbaum Associates, Inc.

Topham, G., Hubbs-Tait, L., Rutledge, J., Page, M., Kennedy, T., Shriver, L., \& Harrist, A. (2011). Parenting styles, parental response to child emotion, and family emotional responsiveness are related to child emotional eating. Appetite, 56 (2), 261-264. http://dx.doi.org/10.1016/j.appet.2011.01.007

Vinden, P. (2001). Parenting attitudes and children's understanding of mind: A comparison of Korean American and Anglo-American families. Cognitive Development, 16 (3), 793-809. http://dx.doi.org/10.1016/S0885-2014(01)00059-4

Wilson, K., \& Prior, M. (2011). Father involvement and child well-being. Journal of Paediatrics and Child Health, 47, 405-407. http://dx.doi.org/10.1111/j.1440-1754.2010.01770.x

Zahn-Waxler, C. (2010). Socialization of emotion: Who influences whom and how? New Directions for Child and Adolescent Development, 2010 (128), 101-109. http://dx.doi.org/10.1002

$\begin{array}{lr}\text { Historial do artigo } \\ \text { Recebido } & 03 / 18 \\ \text { Aceite } & 01 / 19 \\ \text { Publicado } & 08 / 19\end{array}$

